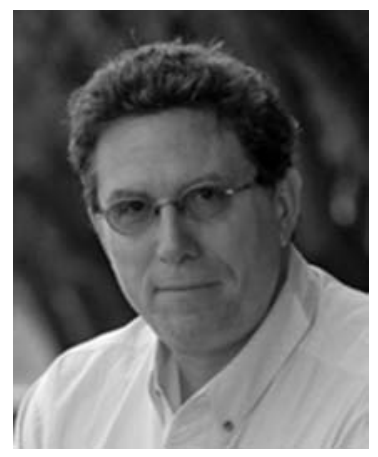

\title{
A Conversation with David Anderson
}

\author{
INTERVIEWER: ANN GOLDSTEIN \\ Senior Editor, Cell Press
}

David Anderson is the Seymour Benzer Professor of Biology at the California Institute of Technology and an Investigator of the Howard Hughes Medical Institute.

Ann Goldstein: More than 10 years ago you switched your research focus from trying to understand neurodevelopment and how stem cells became neurons and glia to studying neuronal behavior. What motivated the switch?

Dr. Anderson: It's a combination of boredom and looking for new sources of excitement and stimulation. I think I do my best work when a field is new and there's more room to flail around and try things before the problem gets very reduced and focused.

Ann Goldstein: What was the most surprising thing you encountered as you made the switch?

Dr. Anderson: How difficult it was and how long it took. It was like trying to change an oil tanker in midcourse. It's not like you can walk into the lab one day and just ask everybody working on development to leave and hire a new group of equally talented people in a new field where nobody knows who you are.

In some ways it was like starting all over again as an assistant professor and having to build a new reputation and a new field from scratch.

The other thing I hadn't anticipated is how long it would take to get the tools we needed to do the kind of experiments I wanted to do-which was to use molecular genetic tools to mark and map and manipulate different populations of neurons.

We worked on developing some of that technology, but it just wasn't there in the mouse, which is why I started working on flies as well, around 2002, because there the technology did exist.

Of course, with the advent of newer techniques like optogenetics in 2005 and more sensitive calcium indicators, that's totally changed. Now the field is as crowded as the stem cell field was when I left it.

Ann Goldstein: And in working with both flies and with mice, you found surprising similarities.

Dr. Anderson: We found similarities at both the circuit level and the molecular level. The fly brain is anatomically completely different from the mouse brain.
Yet - and this is one of the things I find very surprising - in the two systems, the mouse and the fly, using completely independent approaches, we have discovered a small population of neurons that controls both fighting and mating behavior.

In the case of the mouse, it's about 2000 neurons in the hypothalamus. In the case of the flies, it's about three to five neurons in the structure of the brain where the neurons are male-specific. That was a complete surprise. This is still preliminary, but in both cases it seems as if these neurons control state-dependent properties that are associated with these behaviors-levels of arousal, for example - rather than just functioning as switches that determine whether the output is mating or fighting.

That's an example of what seems to be some kind of a circuit motif even though in both systems the structures concerned have no relationship. At the molecular level, I certainly never expected to find genes that were involved in behaviors like mating and fighting and are used in the same way in these two species, but in an unbiased screen recently in flies we turned up a group of cells that release a neuropeptide called tachykinin, which is very important in controlling male-specific aggression in flies.

It turns out that homologs or orthologs of tachykinin in mammals, which are called Substance P or tachykinins, control aggression in rats, in mice, and in cats. There's even a paper from two years ago describing a study of humans by a psychiatrist which showed there was a weak correlation between levels of self-reported aggressiveness and levels of this neuropeptide measured in the cerebral spinal fluid by radioimmunoassay in patients with personality disorders. That's just a correlation, but there are a growing number of examples of neuropeptides that play a conserved role in survival behaviors in vertebrates and invertebrates, like neuropeptide $\mathrm{Y}$, which controls feeding in mammals and in flies, and some evidence that oxytocin, which has been implicated in reproductive behavior in mammals, controls mating in C. elegans as Cori Bargmann has shown.

These behaviors, these survival behaviors, which I call the four Fs - feeding, freezing, fighting, and mating- 
seem to be controlled to a first approximation by molecules, at least neuropeptides, that have a conserved function. Why that should be, I think, is a deep question and the answer is not clear yet, but it was very surprising to me to find that.

Ann Goldstein: Is that the reason why you decided to start with studying aggression?

Dr. Anderson: I started by working on fear because I was interested in emotional behaviors. And fear-certainly learned fear-from studies of Joe LeDoux and others, was one of the best-characterized emotion circuits, certainly in mice.

The problem was it was difficult to get traction on that in flies. We've continued to work on it, but it's still a matter of debate whether the avoidance responses that flies show to something like a looming shadow involve some sort of persistent internal state, that might be likened to a fear-like state, or whether they're just reflexes, stimulus-response reflexes.

I gravitated towards aggression because at the behavioral level there's just no argument about whether flies are fighting or not. They fight and vertebrates fight, and so when we're starting with obvious conservation at the level of the behavior itself, one could use that as a way of really studying, in parallel, what looks like the same generic behavior in the two systems.

Ann Goldstein: You mentioned that the mouse field now has so many great tools that allow you to ask the kind of questions about aggression in mice. What kind of tools do you want to see more of in the future to help push the field a little further?

Dr. Anderson: Let me start with flies because I think that it's to the point now — and the fly people are going to hate me for saying this - that certain things (experiments at the neural circuit level) are currently easier to do in mouse than in the fly.

It is easier to trace the downstream outputs and the upstream inputs that connect to a given population of genetically identified neurons in mice than in flies, because in mice we have tracer viruses, which cannot be used in flies right now, and which can be genetically targeted to cell populations in mice to map upstream and downstream connections.

We have slice electrophysiological recordings where we can stimulate a particular input from a particular location and record from postsynaptic neurons to confirm synaptic connectivity, and the sign of the connection (whether it's excitatory or inhibitory). And we can record and image neuronal activity in awake, behaving animals, even in freely behaving animals. None of those things are currently possible to do in flies.

Gerry Rubin argues that we won't need viral tracers in flies because in five years we'll have a complete connec- tome for the fly brain. That may be true, but I do think that having a way of seeing if the neurons you manipulate functionally are actually active during a particular behavior is really important in flies.

I do think having better tracing techniques in flies would be useful. In mice we have such methods, but many of these viral tracing techniques kill the upstream and downstream neurons they infect.

What we want are viral tracers that label those cells but don't kill them so that when we find the next upstream or downstream cell we can see what happens when we stimulate or inhibit them.

Ann Goldstein: And continue down and up the circuit.

Dr. Anderson: Yes, just move down and up the circuit without having to worry about toxicity. I think that's a really important issue. We also need, and this is really a plea, better ways of automatically quantifying behavior in the mouse. Most of the current methods are based on a single mouse moving around in an arena. The kinds of social behaviors we study happen between mice in their home cage and where we're trying to distinguish mating, fighting, sniffing, scratching, and rearing, which is very difficult to do.

For humans to try to score that on a frame-by-frame basis at 30 frames per second, and do it for hundreds of hours of movies, is rate limiting. When we developed those kinds of techniques with Pietro Perona in flies in 2009, automatic behavior recognition was absolutely transformative. We've now done an unbiased screen for aggression-promoting neurons in flies from 3000 different lines together with Gerry Rubin at Janelia Farm. We could never have done that without automated behavior recognition software.

I think that's an area in which the mouse system is lagging behind. Of course the technique everybody wants, which I think is still a way off, is to do the experiment called "playing the piano." Record and play back. Everybody wants to be able to image a group of neurons in the mouse brain under a particular behavioral condition. Watch the spatiotemporal dynamics of neuronal firing and then play back the same spatiotemporal dynamics of firing using multiplexed optogenetic stimulation, with different colors and single cell activation, to see which aspects of those dynamics are really essential for evoking the behavior.

I think that's going to take a little longer. It's coming, and probably in the next three to five years you'll see examples of that. And then we will need the ability to do these kinds of experiments in more than one brain region at a time. (Relative to a human or a monkey) the mouse brain is very tiny and so to try to put even a microendoscopic lens in the hypothalamus and the amygdala, which are half a millimeter apart, is impossible right now. Further miniaturization is critical. 


\section{$\$_{\text {CSH }}^{\infty}$ Cold Spring Harbor Symposia SYMPOSIA On Quantitative Biology}

\section{A Conversation with David Anderson}

Cold Spring Harb Symp Quant Biol 2014 79: 253-254

Access the most recent version at doi:10.1101/sqb.2014.79.01

\section{License}

Email Alerting Receive free email alerts when new articles cite this article - sign up in Service the box at the top right corner of the article or click here. 